\title{
Research on the Science and Technology Policy Coordination of China's Yangtze River Delta
}

\author{
Binfeng Xu, Wei Song, Xiaopei Gao*, Yu Zhang \\ University of Science and Technology of China, Hefei, China \\ Email: ^gxp0406@mail.ustc.edu.cn,xbf0215@ustc.edu.cn
}

How to cite this paper: Xu, B.F., Song, W. Gao, X.P. and Zhang, Y. (2019) Research on the Science and Technology Policy Coordination of China's Yangtze River Delta. American Journal of Industrial and Business Management, 9, 875-885.

https://doi.org/10.4236/ajibm.2019.94059

Received: February 16, 2019

Accepted: April 12, 2019

Published: April 15, 2019

Copyright () 2019 by author(s) and Scientific Research Publishing Inc. This work is licensed under the Creative Commons Attribution International License (CC BY 4.0).

http://creativecommons.org/licenses/by/4.0/

\begin{abstract}
Science and technology innovation policies have been considered as effective policy tools in promoting regional innovation. This paper used content analysis method to analyze 321 pieces of science and technology policies published from 2006 to 2018. Structures of the policy social networks had been analyzed and discussed, and the main points and emphasis of the science and technology policy system of China's Yangtze River Delta region had been revealed. Results indicated that Shanghai, Jiangsu, Zhejiang and Anhui's science and technology policy system structures were relatively consistent, while the development paths were different, and the content of industrial policy of four provinces had a serious homogenization problem.
\end{abstract}

\section{Keywords}

Yangtze River Delta, Science and Technology Policy, Policy Coordination

\section{Introduction}

In recent years, studies in policy coordination have increased gradually. More and more researchers realized the importance of synergy in policy-making. Lengrand (2003) proposed that science and technology innovation policies are located in the center of government policy, and synergy between innovation policies is very important [1]. Meijers and Stead (2004) argued that policy synergy referred to the management of cross-border issues when formulating policies. Such issues exceeded the responsibilities and authority of individual government or individual government functions and required multiple government functions to work together [2]. Peng Jisheng (2008) proposed that science and technology policy cooperation referred to the process by which the science and technology management department formulated science and technology policies in order to achieve its goals [3]. Ye Dafeng (2015) proposed that the coordina- 
tion of science and technology policies should include the coordination of policy-making body and policy interests, she believed that in the context of China, the formulation of science and technology policies should adhere to the principle of government-led [4]. Zhang Wei (2016) believed that regional science and technology policy coordination referred to the process of achieving coordination in different types of science and technology policies issued by different policy-making body in the region [5].

In the aspect of scientific and technological policy content analysis, Su Jingqin (2012) took Dalian as an example, using content analysis method to analyze the similarities and differences of policy focus of science and technology policy at the national and regional levels, and drawn the social network of science and technology policies of two levels with Ucinet software [6]. Huang Jing (2014) introduced quantitative statistical and multidimensional scaling analysis into the research field of local scientific and technological achievements transformation policy, and took 239 local scientific and technological achievements transformation policies as samples to analyze the time distribution, type distribution, geographical distribution of the sample policies [7].

It can be seen that studies on the coordination of science and technology policy have made great progress in both qualitative and quantitative research ways, in terms of its connotation and scope. However, the previous studies also have the following shortcomings: 1) Studies on the effectiveness of science and technology policy is insufficient, basically based on the science and technology policy before 2012. 2) Research works were basically based on the current situation of the policy, only a few studies have explored the development trend of the policy, which makes the corresponding policy recommendations lack of forward-looking.

\section{Coordination Research on Science and Technology Policy Content in the Yangtze River Delta Region}

\subsection{Research Design}

Policies released by the government directly reflected the means, purposes, focus-points and values of the authority. This paper used content analysis method as the main research method. The large contents of policy texts have been transformed into quantitative data. The Microsoft Excel software was used to clean up and statistically analyze those data. A keywords matrix was formulated, and the social networks of S\&T policies had been depicted by UCINET software. In the social network map, the centralities and main focus points of policies had been revealed. Then, the policy intentions and development of the government could be explored in the S\&T policy system.

\subsection{Data Collection}

In order to compare the policy focus points among different areas, the data should reflect the structure of the policy system in China's Yangtze River Delta region. Based on the previous research by Liu Fengchao and Sun Yutao (2007) 
[8], and 3 important S\&T policy documents: Decision on the reform of the science and technology system (1985), Decision on accelerating scientific and technological progress (1995) and National Medium- and Long-Term Science and Technology Development Plan (2006 to 2020), the development of Chinese S\&T policies had been assigned into 4 stages of period: Recovery Stage (1978 to 1985), Self-improvement Stage (1985 to 1995), Rejuvenating Stage (1995 to 2006) and Innovation Stage (2006 to date). The Innovation Stage matches the policy time period of National Medium- and Long-Term Science and Technology Development Plan (2006 to 2020).

In this paper, the S\&T policies in Yangtze River Delta region in the Innovation Stage time periods had been collected. Policy documents released by the local government in Shanghai, Jiangsu, Zhejiang and Anhui had been gathered from Peking University Policy and Law Database, the websites of the local government, People's Congress and CPPCC in various administrative levels, and the websites of functional departments in local government including Science and Technology Department, Intellectual Property Office, Development and Reform Commission, Department of Education, Finance Department, Financial Office, Economic and Information Commission and Industry and Commerce Bureau. To preserve the integrity and reliability of data, the policy documents should comply with the following standards: First, the documents should be released directly by the local government or its functional departments in the Yangtze River Delta region. Second, the content of policy documents should be directly related with innovation. Third, the policy documents should belong to one of the following categories: the government regulations, planning, proposals etc. 321 policy documents had been selected from the raw data, 72 documents from Shanghai, 69 documents from Jiangsu, 97 documents from Zhejiang and 83 documents from Anhui. Those documents would be the researching subject of this paper. It's worth noting that most policy documents released jointly by government in more than one province did not comply with the third standard. Therefore, those policies would not be studied in this paper.

\subsection{Data Processing}

The contents of S\&T policy documents were the subject of this study. Key words about S\&T innovation have been selected from those documents, information about policy purpose and means were extracted and quantified.

In order to extract as many policy key points as from the documents, the number of key words and policy categories were not limited. Key words that had similar policy point were combined as one, like "Technology and financial synergy" and "Technology and finance combination". The frequency of occurrence of extracted key words was calculated and analyzed using Microsoft Excel. Key words that existed in more than 50 documents were selected from the data. And those whose occurrence frequency relatively low were eliminated from the study to avoid interference.

This process had been applied on the 72 documents from Shanghai, 69 docu- 
ments from Jiangsu, 97 documents from Zhejiang and 83 documents from Anhui. 52 key words from Shanghai, 59 key words from Jiangsu, 51 key words from Zhejiang and 57 key words from Anhui had been generated from data.

Those key words were used to build the key words co-occurrence matrix in the four regions, with a $52 \times 52$ matrix in Shanghai, a $59 \times 59$ matrix in Jiangsu, a $51 \times 51$ matrix in Zhejiang and a $57 \times 57$ matrix in Anhui. Numbers in the diagonal indicated the frequency of occurrence of that key word. Numbers in A row B column indicated the co-occurrence frequency of those two key words. Mapping those matrixes in Netdraw, the network of policy key word co-occurrence was presented in Figures 1-4. Notes in the map represented certain policy point. Lines between notes represented the co-occurrence relationship, and the thickness of the lines represented the frequency of co-occurrence.

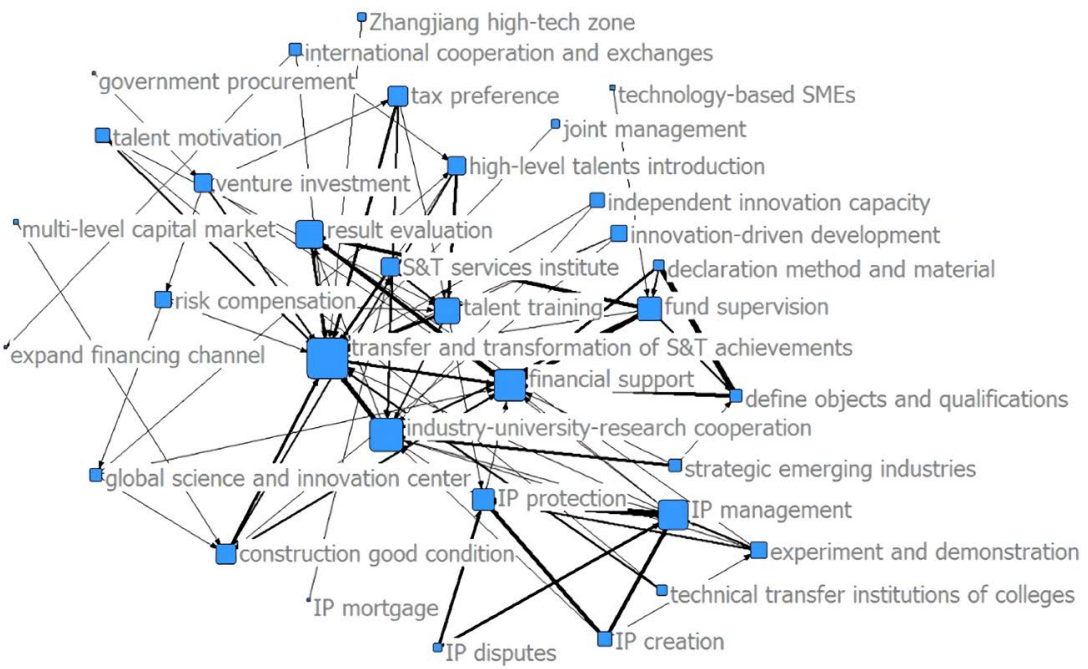

Figure 1. S\&T policy social network map of Shanghai city.

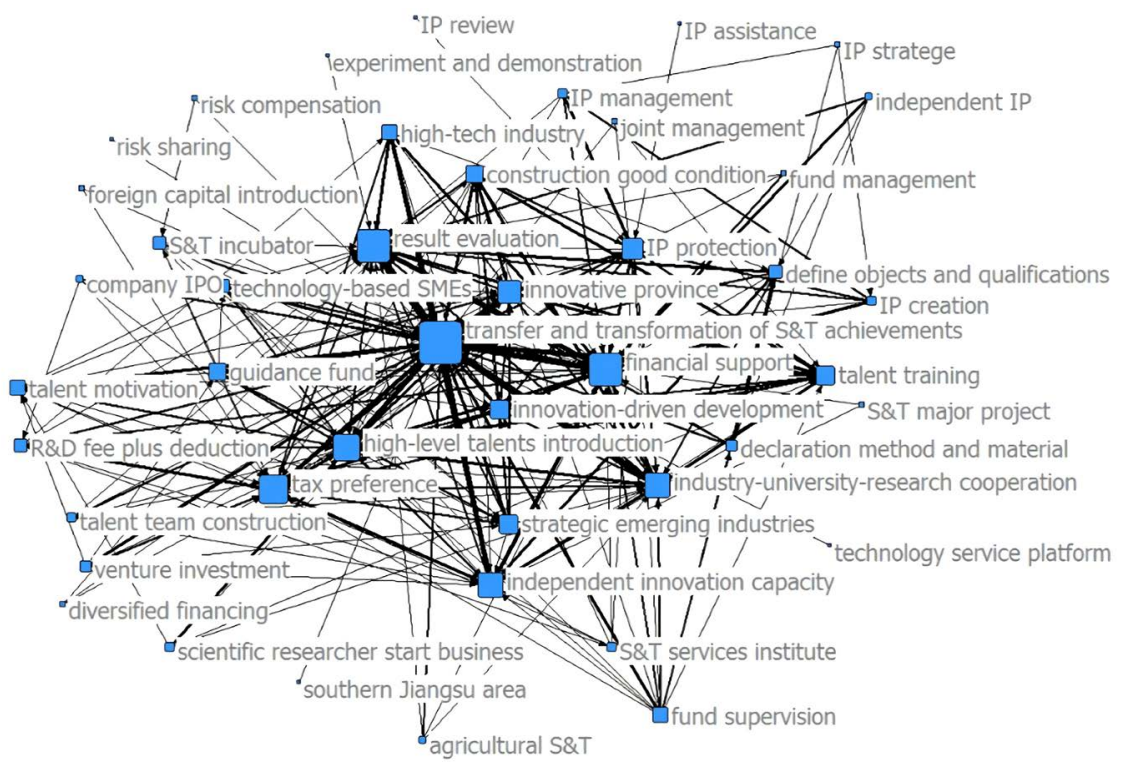

Figure 2. S\&T policy social network map of Jiangsu province. 


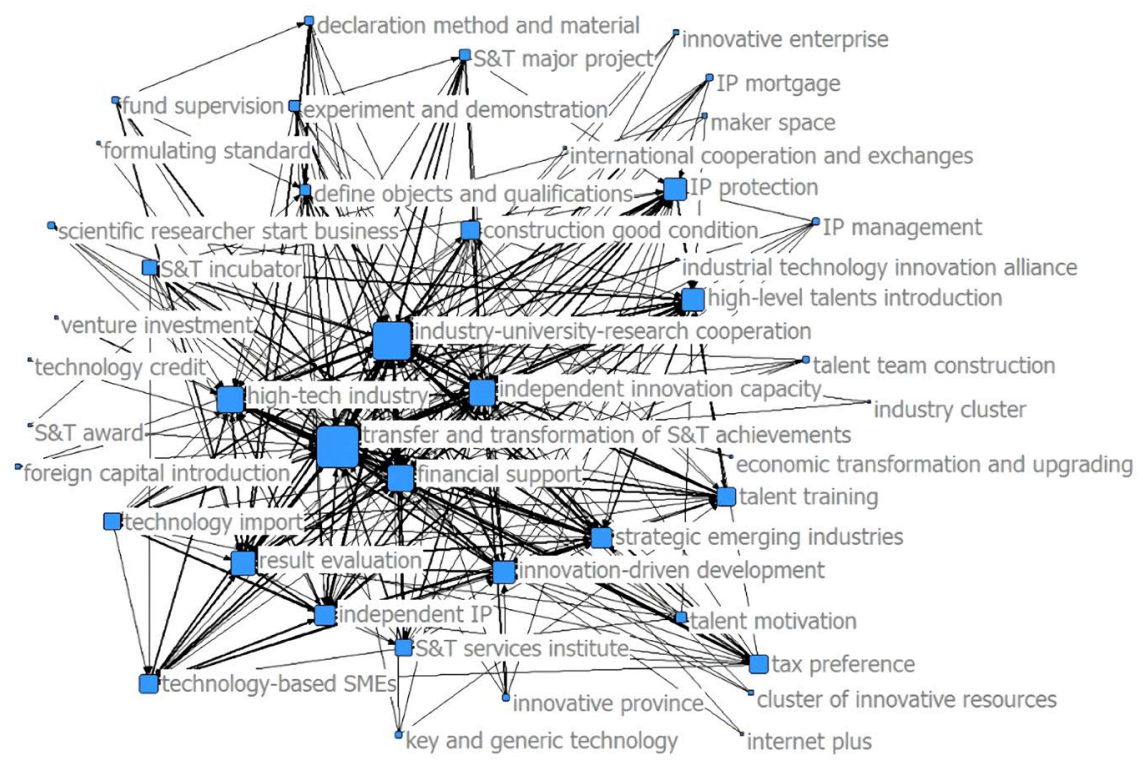

Figure 3. S\&T policy social network map of Zhejiang province.

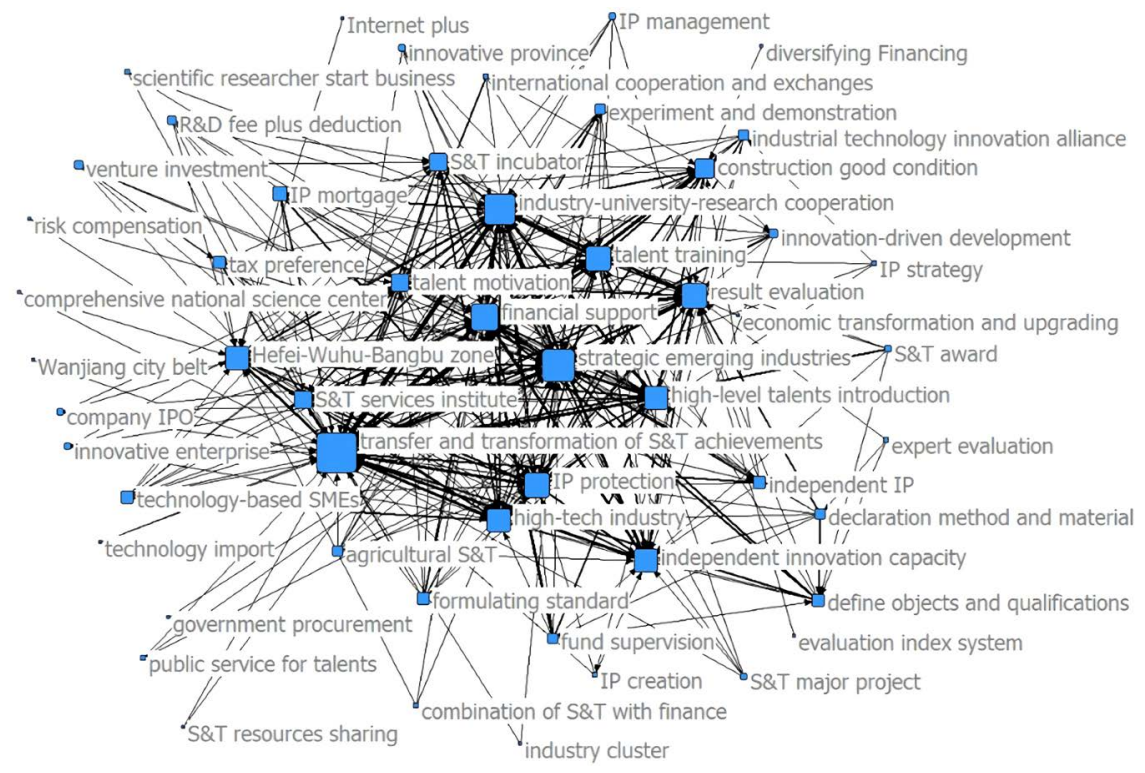

Figure 4. S\&T policy social network map of Anhui province.

\subsection{S\&T Policy Social Network Mapping}

Figure 1 is a social network graph consists of the high-frequency vocabulary of Shanghai's science and technology innovation policy. Keywords such as industry-university-research cooperation, transfer of scientific and technological achievements, financial support, and personnel training are more important in the network. This means science and technology policy of Shanghai city mainly focused on industry-university-research cooperation, transformation of scientific and technological achievements, financial support, talent training, and the introduction of high-level talents. This also reveals that most governments pay more attention to the process of formulating science and technology policies. 
Among those keywords, the transfer of scientific and technological achievements and the industry-university-research cooperation, financial support, talent cultivation and the creation of a good environment are relatively close, indicating that Shanghai encouraged enterprises to cooperate with universities and scientific research institutions with strong innovation capabilities and more intensive talents, and provided adequate protection from the aspects of capital and environment. In terms of intellectual property, intellectual property protection is closely related to intellectual property management, intellectual property creation and use, and intellectual property disputes. This reveals that Shanghai is committed to building a comprehensive intellectual property system to safeguard and support the process of technological innovation. At the edge of the policy network, there are keywords such as multi-level capital markets, international cooperation and exchanges, and Zhangjiang High-tech Zone. Although the attention on these keywords is not very high, it also reflects the trend of diversified development in the field of science and technology policy formulation.

As shown in Figure 2, In Jiangsu Province, the science and technology policy system mainly focused on transformation of scientific and technological achievements, the construction of innovative province, and the innovation-driven development. Among them, the transfer of scientific and technological achievements and innovation-driven development, strategic emerging industries, high-level talent introduction, industry-university-research cooperation, technology-based SMEs, and talent training are more closely linked. Those results reveals that science and technology policy system of Jiangsu province mainly focused on the transformation of scientific and technological achievements, the construction of talent team, the cooperation of industry-university-research in strategic emerging industries and high-tech industry. Compared with the previous policy focus on foreign investment and technology introduction, the current science and technology policy has focused more on independent innovation capability and intellectual property. In addition, the South Jiangsu region, R\&D expenses plus deductions, tax preference, talent incentive and technology service institution are also involved in the policy system, reflecting that the Jiangsu province's science and technology policy system had a clear focus and comprehensive coverage.

As shown in Figure 3, similar to Jiangsu Province, Zhejiang Province's science and technology policy is centered on key words such as transformation of scientific and technological achievements, industry-university-research cooperation, high-tech industry, independent innovation capabilities, and financial support. Among them, the transformation of scientific and technological achievements has strong links with industry-university-research cooperation, high-tech SMEs, high-tech industries, independent innovation capabilities, and independent intellectual property. This reflects that the goals of Zhejiang's science and technology policy system were mainly to introduce high-level talents and technologies, strengthen the cooperation between private enterprises, universities and scientific research institutions, cultivate the development of high-tech industries and strategic emerging industries, produce a group of independent intellectual 
property rights with market competitiveness, realize the transfer and transformation of scientific and technological achievements and drive economic and social development with innovation. At the edge of the policy network, key words with strong local characteristics such as venture investment, social capital, technology credit, foreign capital introduction, "internet+", industrial chain, and marine technology are also involved, which not only indicate that the focuses in the Zhejiang Province's science and technology policy system were clear, but also reflected the social and economic characteristics of Zhejiang province.

As shown in Figure 4, in Anhui Province's science and technology policy network, the focus is on the transfer and transformation of scientific and technological achievements, independent innovation capability, strategic emerging industries, industry-university-research cooperation, intellectual property protection, high-level talent introduction and Hefei-Wuhu-Bengbu independent innovation demonstration zone. The economic and social development of Anhui province is weaker than Shanghai, Jiangsu and Zhejiang province. However, Anhui Province has a clear development logic, it gathered the limited resources in Hefei-Wuhu-Bengbu independent innovation demonstration zone and strategic emerging industries, which it has strong independent innovation capabilities. In the periphery of the policy social network, there are also keywords such as Wan-jiang urban belt, researcher's entrepreneurship, the comprehensive national science center and the sharing of scientific and technological resources, which fully reflects the status of Anhui Province's innovation.

\section{Result Analysis and Discussion}

Through the analysis of the contents of science and technology policy system in the three provinces and one city of the Yangtze River Delta, this paper found out that three features existed: the science and technology policy architecture were consistent, the regional development paths of three provinces and one city were different and the content of industrial policy of three provinces and one city had a serious homogenization problem.

\subsection{The Science and Technology Policy Architecture Were Consistent}

Results from the regional innovation network map indicated that in the Yangtze River Delta region, S\&T transferring and transformation, independent innovation capacity building, human resource establishment, financial support had been the main policy point and purpose of these regions from 2006 to 2018. To achieve these policy goals, those regions had employed innovation-based policy tools such as Major S\&T Projects, Industry-University-Research Cooperation, Industrial Technology Innovation Alliance, S\&T rewards, Innovative Resource Agglomeration, S\&T Resource Sharing. Other kind of policy tool had also been applied, including intellectual property-based policy tools such as IP Law Enforcement, IP Judicial Protection, IP Right Assistance, IP Warning and IP Alliance; human resource and S\&T transferring-based policy tools such as High-level tal- 
ent introduction, Talent Development, HR Public Services, Overseas R\&D institutions acquisition, International Technology transferring; capital investment-based policy tools such as pure capital input, Special subsidy, Tax incentives, R\&D expenditure plus deduction, Diversified financing, Intellectual property pledge, Risk compensation, IPO Promotion, Equity incentive; university and research institution-based policy tool such as Incubation, Researchers Entrepreneurship, IP right reforms, IP Shareholding.

\subsection{The Regional Development Paths of Three Provinces and One City Were Different}

Generally speaking, the high-frequency words of science and technology policies in the three provinces and one city in the Yangtze River Delta region all include keywords such as "transfer and transformation of scientific and technological achievements", “industry-university-research cooperation", “strategic emerging industries" and "independent innovation capability", but other important keywords were different. The three provinces and one city in the Yangtze River Delta determined science and technology goals based on local economic and social development situation. This leads to different regional development paths.

Shanghai's science and technology policy focused more on industry-university-research cooperation, it promoted the process of transfer and transformation of scientific and technological achievements on the basis of the development of original technology. Geographically, it focused on Zhangjiang High-tech Zone and Shanghai Free Trade Zone.

The science and technology policy of Jiangsu Province mainly emphasized the transfer and transformation of scientific and technological achievements and the development of strategic emerging industries. The region mainly emphasized the northern Jiangsu area and independent innovation demonstration region in southern area of Jiangsu. It is pointed out that science and technology should be used to promote the economic transformation and upgrading in the northern Jiangsu region, and it also emphasized to accelerate the construction process of independent innovation demonstration region in southern area of Jiangsu through promoting the independent innovation capability and the transformation of scientific and technological achievements.

In addition to focusing on the transfer of scientific and technological achievements, the policy objectives of Zhejiang Province were mainly focused on the private economy and the Internet economy. On the one hand, it is necessary to strengthen the independent innovation capacity building of SMEs, help them become high-tech enterprises with scientific and technological services, and build industrial technology innovation alliances to enhance the core competitiveness of related industry. On the other hand, it is committed to achieving cross-border integration of "Internet+" and manufacturing, agriculture, and logistics to promote the development of traditional industries.

Anhui Province had strong scientific and technological innovation capability, but its economic and social development is not as good as Shanghai, Jiangsu and 
Zhejiang province. Therefore, the science and technology policy of Anhui Province reflected the characteristics of the accumulation of innovative resources. Firstly, the construction of the Hefei-Wuhu-Bengbu independent innovation demonstration zone was highly valued by the government departments. The government proposed the accumulation and sharing the scientific and technological resource, promoting the improvement of original independent innovation capability through basic science and technology development. Secondly, the construction of the Wanjiang city belt is positioned to undertake the transfer industry form Chinese coastal regions, and use further technological innovation to drive regional economy and development.

Different science and technology innovation policy systems would lead to different development paths in Shanghai, Jiangsu, Zhejiang and Anhui provinces. Shanghai paid more attention to industry-university-research cooperation, which will produce more original basic knowledge and technology, and technology products would also be more expressed as technology services than physical products. Jiangsu's science and technology innovation policy system had played a good role in promoting the agglomeration and development of its strategic emerging industries, and improved the current situation of uneven development in the north and south. Jiangsu's science and technology innovation policy system played a good role in promoting the agglomeration and development of its strategic emerging industries, and improved the current situation of uneven development in the north and south. Anhui Province could create industrial clusters through industrial transfer, but the policy of high concentration of innovative resources may also lead to uneven development in the province.

\subsection{The Content of Industrial Policy of Three Provinces and One City Has a Serious Homogenization Problem}

From the analysis of the science and technology policies social network in Shanghai, Jiangsu, Zhejiang and Anhui province strategic emerging industries is one of the key point policies that directly affect the future development of Yangtze River Delta. Therefore, this paper believed that it is necessary to conduct in-depth research on strategic emerging industry policies. After analyzing the strategic emerging industries policy contents of the three provinces and one city in the Yangtze River Delta, results showed that policy contents were similar, especially in the field of segmentation industry selection and technological innovation breakthrough areas.

This showed that there was a lack of regional overall system design in the regional innovation industry policies of the three provinces and one city in the Yangtze River Delta region and there was need to strengthen the complementarity. The homogenization of industrial policies will directly affect the homogenization of supporting policies such as talent policy and technology introduction, which will easily lead to bad competition in the future industrial structure of the Yangtze River Delta region, and form a "zero-sum game" phenomenon, and result in repeated investment in innovation funds and infrastructure construction. 
It may cause a new round of overcapacity in the future and increase coordination expenditures in the region.

\section{Conclusions and Recommendations}

Through the analysis of the science and technology policy system of the three provinces and one city in the Yangtze River Delta, results revealed that the Yangtze River Delta science and technology innovation policy system had been initially established and continuously improved, but in content of industrial policy of four provinces, there has a serious homogenization problem. It showed that there is a lack of effective coordination mechanisms among the provinces' policy-making organization. Therefore, this paper proposes the following suggestions:

1) Shanghai city, Jiangsu, Zhejiang and Anhui Province should establish a cooperative mechanism for inter-regional policy, pay attention to the differences and complementarities of its industrial policy system, to give full play to the synergies in regional innovation and development. Specifically: First, in the choice of future industries, we can consider dislocation development, achieve win-win through the cooperation and exchange of innovation entities; second, in the stage of industrial development, they should consider sub-regional development. For example, Shanghai city and Anhui province have strong original knowledge and basic technology research and development capabilities as a comprehensive national science center, while Jiangsu Province and Zhejiang Province has more complete market-oriented mechanism construction to improve applied technologies and realize the transfer of scientific and technological achievements. Therefore, it is recommended to establish a phased technology development and technical compensation mechanism in order to promote regional coordinated development.

2) In the process of formulating science and technology policies, the construction of coordination mechanisms and communication mechanisms for science and technology management departments in all provinces should be strengthened, and the rotating secretariat of the joint meeting of the Science and Technology Department of the provinces to be responsible for the daily operation of the joint meeting should be established. In terms of communication mechanism, it is recommended to establish an information sharing platform for inter-departmental coordination to strengthen interaction and coordination among provinces, cities and departments, and improve the operability of the implementation of science and technology policies.

\section{Research Prospect}

This paper studied the science and technology innovation policies in the Yangtze River Delta region of China, and analyzed the policy content in a social network analysis method. It revealed the characteristics and shortcomings of Shanghai, Jiangsu, Zhejiang and Anhui science and technology innovation policy system, 
which could provide reference for policy makers in these provinces. However, the scope of policy analysis of the paper is limited in science and technology innovation policies, future studies could include industrial policies to better study the relationship between their leading industries and technological innovation.

\section{Conflicts of Interest}

The authors declare no conflicts of interest regarding the publication of this paper.

\section{References}

[1] Lengrand, L. (2003) Innovation Tomorrow: Innovation Policy and the Regulatory Framework: Making Innovation and Integral Part of the Broader Structure Agency. Office for Official Publications of the European Communities, Brussels.

[2] Meijers, E. and Stead, D. (2004) Policy Integration: What Does It Mean and How Can It Be Achieved? A Multi-Disciplinary Review. Berlin Conference on the Human Dimensions of Global Environmental Change: Greening of Policy-Interlinkages and Policy Integration.

[3] Peng, J.S. (2000) Chinese Technology Collaborative Innovation. Economic Science Press, Peking.

[4] Ye, D.F. (2015) Cooperative Governance: New Exploration of the Governance Model of Policy Conflict. Management World, No. 6, 172-173.

[5] Zhang, W., Fei, X.Y. and Fang, H. (2016) Design and Construction of Evaluation Index System of Regional Innovation Policy Based on a Multi-Perspective. Science \& Technology Progress and Policy, No. 1, 142-147.

[6] Su, J.Q., Li, X.A. and Xu, X.A. (2012) Comparative Analysis on Technology Innovation Policy of State and Local Based on Content Analysis. Science of Science and Management of S. \& T, No. 6, 15-21.

[7] Huang, J. (2014) Research on the Transformation Policy of Science and Technology Achievements in Chinese provinces. Science \& Technology Progress and Policy, No. 13, 103-108.

[8] Liu, F.C. and Sun, Y.T. (2007) The Course of, Trend in and Proposal for Evolution from S\&T Policies to Innovation-Based on the Empirical Analysis of China's 289 Innovation Policy Documents. China Soft Science, No. 5, 34-42. 